\title{
Research on the influence law and suppression methods of seismic wavelet time-varying nature on seismic data processing
}

\author{
Peng Zhang, Yongshou Dai, Manman Zhang, Rongrong Wang \\ College of Information and Control Engineering \\ China University of Petroleum \\ Qingdao, China \\ upczhangpeng@163.com
}

\begin{abstract}
To adjust to the development of dynamic prediction of oil and gas reservoir, exploration of subtle reservoir, exploration and development of thin interbeds, processed seismic profiles are required to be of high signal to noise ratio, high resolution and high fidelity. While the time-varying wavelet in seismic data is an important factor affecting the quality of seismic profiles, for the purpose of improving the effects of seismic data processing, the influence law of wavelet time-varying nature is necessarily to be analyzed and researched, so that the timevarying wavelet can be suppressed based on more targeted methods. In the paper, the formation mechanism of time-varying nature is studied, and the influence law of time-varying wavelet is analyzed in detail, which is different in various links of seismic data processing, including amplitude compensation, pre-stack deconvolution, post-stack deconvolution and migration. Meanwhile the methods and application effects for suppressing wavelet time-varying nature in every link are summarized, and the advantages and disadvantages of these approaches are concluded through analysis and comparison, so that the development direction of researching more effective methods for suppressing or eliminating wavelet time-varying nature in the future is pointed out.
\end{abstract}

Keywords—seismic data processing; seismic wavelet; timevarying nature; influence law; suppression methods

\section{INTRODUCTION}

The goal of seismic data processing is to improve the SNR, resolution and fidelity of seismic reflection wave. The traditional methods are based on the assumption that the seismogram is stationary. While the seismic wavelet in practical data is of time-varying, with the high frequency component and the phase characteristics changing with time. Because of the severer impact on the seismic data processing, suppression of wavelet time-varying nature is significant. Some researchers tried to eliminate the influence of wavelet timevarying nature indirectly by means of high frequency compensation [1]. The others committed to extract timevarying seismic wavelet from the seismic record directly and eliminate its impact effectively through wavelet deconvolution [2]. However, there is no deep analysis of the influence law on different links of seismic data processing so that the practical application of existing methods does not achieve good effects.
In order to provide references for further research on suppressing or eliminating wavelet time-varying nature more effectively, in this paper, considering the Earth's absorption and attenuation effect mainly, the influence law of wavelet timevarying nature on seismic data processing is analyzed and studied, and then the existing mainstream approaches of timevarying wavelet suppression and their advantages and disadvantages are summarized aimed at different processing links.

\section{FORMATION MECHANISM OF WAVELET TIME-VARYING NATURE}

Seismic wavelet is time-varying, with the waveform changing with time. The following points are the main influence factors of wavelet waveform.

(1) The waveform of source. It is affected by p-wave velocity, shear wave velocity, the explosion cavity radius of the shotpoint. The energy and frequency component of source wavelet at different excitation locations are also different.

(2) The absorption effect of the Earth cause high frequency attenuation and phase distortion of seismic wavelet, so that the waveform of wavelet changes.

(3) The influence of the detector-earth coupling system. It depends on the fastness degree and the contact area between the detector and the ground. When the effect of embedment geophone is not satisfactory, coupling effect may cause the wavelet phase and the high frequency components changing.

These suggest that the time-varying wavelet is produced in the process of seismic wave excitation, transmission and reception, with the waveform, bandwidth, phase changing with time. Among them, the time-varying nature, produced in the process of excitation and reception, can only be controlled slightly by selecting suitable excitation mode and detector type, can't be eliminated effectively. And the time-varying nature, come from the process of transmission, may affect the effects of seismic data processing seriously [3].

Influenced by the impact of time-varying nature, the seismogram is non-stationary [4], which can be expressed by 


$$
s(t)=\iint \omega(f) \alpha_{Q}(\tau, f) r(\tau) \exp [2 \pi i f(t-\tau)] d f d \tau
$$

Where $s(t), \omega(f)$ and $r(\tau)$ are the seismogram, the Fourier transform of seismic wavelet and the reflection coefficient sequence respectively. And $\mathrm{Q}$ is the quality factor, $\alpha_{Q}(\tau, f)$ is the transfer function of the stratigraphic absorption effect, as shown in the following formula

$$
\alpha_{Q}(\tau, f)=\exp (-\pi f \tau / Q)
$$

Therefore, it is required urgently to analyze the influence law of wavelet time-varying nature on different links of seismic data processing, and research on the effective suppression or elimination methods of the time-varying wavelet.

\section{ANALYSIS ON THE INFLUENCE LAW OF WAVELET TIME- VARYING NATURE}

Traditional seismic data processing methods assumed that the seismic wavelet is time-variant, namely the seismic record is stationary. While actually, the seismic wavelet appears the characteristic of dynamic attenuation in the process of transmission because of the absorption effect of viscoelastic medium. The time-varying nature may affect every link of seismic data processing causing unable to get high quality seismic profiles [5].

It is necessary to do amplitude compensation by means of correcting a variety of factors that cause amplitude changing, so that the reflection ability of the reflection interface can be reflected accurately. However, due to the wavelet time-varying nature not only performs in the change of amplitude energy, but also in the phase distortion, the quality of seismic data remains to be improved after amplitude compensation.

Pre-stack deconvolution aims to improve the resolution of seismic data and weaken the impact of Earth filtering. As we know, seismic wavelet is the main body of deconvolution. If there is no effective pre-stack deconvolution methods to eliminate the time-varying nature, the time-varying wavelet may affect the effect of deconvolution seriously.

The process of stack is to suppress the random disturbance and improve the signal-to-noise ratio of seismic data. But the seismic events can't be corrected satisfactorily and the waveform is not unified after dynamic correction, static correction and residual moveout correction, due to the timevarying nature. This may damage the high frequency components seriously.

Because of the low-pass filtering effect in the process of stack, the frequency bandwidth of wavelet which has been broadened turns to be narrow again, causing that there remains the time-varying nature affecting the effect of post-stack deconvolution and the accuracy of inversion results.

The medium velocity, dominant frequency of seismic wavelet and aperture angle are the main parameters that affect the horizontal resolution of migration imaging. So the wavelet time-varying nature would cause a large difference in the amplitude and phase of upcoming and down-going wave field, leading to imaging in the wrong position.

In conclusion, the seismic wavelet with time-varying nature acts on every link of seismic data processing, affecting the quality of seismic profiles after processing. Yet the influence of wavelet time-varying nature on different links is different, it is required to suppress the time-varying wavelet by means of various methods aimed to different links, from different angles.

\section{SUPPRESSION OR ELIMINATION METHODS OF WAVELET TIME-VARYING NATURE}

The time-varying wavelet has a significant influence on the actual effect of seismic data processing and the accuracy of seismic interpretation. It is necessary to explore the suppressing or eliminating methods of wavelet time-varying nature aimed at each link.

\section{A. Methods in Amplitude Compensation}

Mainstream methods for amplitude compensation include spectrum whitening, inverse $\mathrm{Q}$ filtering and time-frequency compensation.

\section{1) Spectrum whitening}

Spectrum whitening can achieve frequency compensation through broadening the amplitude spectrum of seismic signal. But the compensation ability of the method is limited because of the amplitude-only filter and the assumption of white noise reflection coefficient.

\section{2) Inverse $Q$ filtering}

Inverse $\mathrm{Q}$ filtering can not only compensate amplitude energy, but also correct phase. The method was proposed by Hale using series expansion for high frequency compensation [6]. The earth filtering factor is

$$
S(f)=\exp \left\{-\frac{\pi t}{Q}[f+i H(f)]\right\}
$$

Where $\mathrm{f}, \mathrm{t}$ and $\mathrm{Q}$ are the frequency, travel time and quality factor respectively. $\mathrm{H}$ is the Hilbert transform. If $G(f)=f+i H(f)$, the above formula can be shown as

$$
S(f)=\exp \left\{-\frac{\pi t}{Q}[G(f)]\right\}
$$

The process of stratigraphic absorption can be expressed by

$$
X(f)=Y(f) S(f)
$$

Where $Y(f)$ is the seismic wave field regardless of attenuation, and $X(f)$ is the seismic wave field with attenuation. And then, the inverse $Q$ filtering can be expressed as following 


$$
X(f)=Y(f) S(f)^{-1}
$$

Where $S(f)^{-1}=\exp \left\{\frac{\pi t}{Q}[G(f)]\right\}$ is the factor of inverse Q filtering.

However, the premise of the approach is that the $\mathrm{Q}$ value is known, which is time-varying and hard to be obtained precisely.

3) High frequency compensation

The principle of high frequency compensation is

$$
u_{1}(t)=I_{i}(t) * u_{i}(t)
$$

Where $u_{i}(t)$ is the down-going wave field received by the $i^{\text {th }}$ detector, $u_{1}(t)$ is the wave field of initial wavelet, and $I_{i}(t)$ contains the attenuation value of different frequencies.

High frequency compensation based on time-frequency is very popular in recent researches because the time-frequency analysis methods are able to provide joint distribution information of time and frequency domain, and describe the relationship of the signal frequency changing with time. Wavelet transform, $\mathrm{S}$ transform and generalized $\mathrm{S}$ transform are the main methods used for compensation [7,8].

\section{B. Methods in Pre-stack Deconvolution}

It is required to suppress the wavelet time-varying nature in pre-stack deconvolution for the goal of improving the resolution of seismic data [9].

\section{1) Surface consistent deconvolution}

Surface consistent deconvolution is suitable for pre-stack processing, eliminating the surface factors affecting the wavelet. It can improve the SNR of seismic data, suppress the seismic wavelet. But the method is based on the assumption that the wavelet is minimum phase, which is not real. And the noise interference energy in pre-stack data is so strong that the wavelet time-varying nature can't be suppressed completely.

\section{2) Time-varying Wiener deconvolution}

Time-varying Wiener deconvolution is an amplitude-only deconvolution method for processing the problem of wavelet time-varying nature in the process of pre-stack. The precondition is that the phase of seismic wavelet is corrected accurately by phase-only inverse Q filtering. However, inverse $\mathrm{Q}$ filtering can't correct the phase of time-varying wavelet precisely because of the limitation of method itself [10], the effect of time-varying Wiener deconvolution is limited either.

The analyses above suggest that the processing effect of existing methods is not satisfactory. There remains the timevarying nature of seismic wavelet affecting the subsequent links.

\section{Methods in the Process of Stack}

Suppression of wavelet time-varying nature aimed at the process of stack, considering the influence of time-varying nature on the resolution and SNR of seismic data, is to improve the quality of seismic profiles. The suppressing methods include the frequency domain stack and the stretching removal. But they are limited due to the influence of velocity analysis, dynamic and static correction.

\section{Methods in Post-stack Deconvolution}

There would be an inaccurate inversion result because of the wavelet time-varying nature, so the suppression of timevarying wavelet is the emphasis problem that is necessary to be solved in post-stack deconvolution.

\section{1) Piecewise stationary deconvolution}

The idea of the method is to divide the non-stationary seismogram into several segments, so that each segment is approximately stationary. And then, eliminate the influence of seismic wavelet by means of stationary deconvolution methods in each segment. So, selection of piecewise methods is the key point.

The traditional method which is based on overlapping segmentation, is only suitable for the case of non-stationary component changing with time slowly, without considering the actual characteristics of the seismic record. An adaptive segmentation method is developed by L.L. Wang, J.H. Gao, W. Zhao and X.D. Jiang [11]. The self-adaptability manifests as the length of each segment adjusting automatically with the characteristics of seismic record. If a certain constraint is satisfied, the length of data in each segment and the number of segment are determined.

\section{2) Spectrum modeling deconvolution}

Time-frequency spectrum modeling is based on timefrequency analysis methods which is a powerful tool for analyzing non-stationary signal. The time-varying amplitude spectrum can be estimated and then be eliminated at every time point based on the assumption that the amplitude spectrum is smooth.

An improved generalized $S$ transform was proposed to make time-frequency transform [12], using the window function

$$
G(t, f)=\frac{1}{\sqrt{2 \pi} q|f|^{p}} \exp \left(-\frac{t^{2}}{2 q^{2} f^{2 p}}\right)
$$

Where $\mathrm{p}, \mathrm{q}$ are adjustment factors that are greater than zero respectively. And the time-frequency spectrum $S(\tau, f)$ of seismogram $s(t)$ is expressed by

$$
S(\tau, f)=\int_{-\infty}^{\infty} s(t)\left\{\frac{1}{\sqrt{2 \pi} q|f|^{p}} \exp \left(\frac{-(\tau-t)^{2}}{2 q^{2} f^{2 p}}\right) \times \exp (-i 2 \pi f t)\right\} d t, f \neq 0(9)
$$

And then the amplitude spectrum of seismic record in every moment can be obtained when time $\tau$ is fixed. If there is a hypothesis that the amplitude spectrum of seismic wavelet is smooth and the amplitude spectrum of reflection coefficient sequence is oscillating accordingly, the experience formula of 
the amplitude spectrum of seismic data was summarized as following

$$
W(f)=|f|^{k} \exp \left(\sum_{n=0}^{N} a_{n} f^{n}\right)
$$

Where $\mathrm{k}$ is a constant, $\mathrm{N}$ is the order, $\mathrm{a}_{\mathrm{n}}$ is the polynomial coefficient about f, and $0<k<3,2<N<7$ generally [13].

B.W. Tang, B. Zhao, Y.H. Wu and H.Q. Li [14] improved the spectrum modeling and proposed the quadratic spectral modeling on the basis of above. Non-stationary seismogram can be written as

$$
x(t, \tau)=w(t, \tau) * r(t)
$$

By making the time-frequency transform on both sides of the equation, the relationship of amplitude spectrum can be shown as follow

$$
A_{x}(t, f)=A_{w}(t, f) A_{r}(t, f)
$$

Where $A_{x}(t, f), A_{w}(t, f)$ and $A_{r}(t, f)$ are the timefrequency amplitude spectrum of seismogram, seismic wavelet and reflection coefficient sequence respectively. Make Fourier transform on the above expression

$$
A_{x}^{(2)}(t, f)=\frac{1}{2 \pi} A_{w}^{(2)}(t, f) * A_{r}^{(2)}(t, f)
$$

Where $A_{x}^{(2)}(t, f), A_{w}^{(2)}(t, f)$ and $A_{r}^{(2)}(t, f)$ are the Fourier transform of $A_{x}(t, f), A_{w}(t, f)$ and $A_{r}(t, f)$, namely quadratic spectral. If the amplitude spectrum of seismic wavelet is assumed low-frequency, and the time-varying wavelet is able to be extracted by low-pass filtering and eliminated by deconvolution.

The characteristic of the wavelet amplitude changing with time can be described accurately based on the method, with considering the wavelet time-varying nature adequately. But the actual application of the method is restricted because of unable to solve the phase problem.

\section{E. Methods in Migration}

Migration include pre-stack migration and post-stack migration. In pre-stack migration, inverse Q filtering is applied to compensate the high frequency attenuation before migration, and Kirchhoff method is used in the process of migration. The precondition of post-stack migration is that the time-varying wavelet has been eliminated. Therefore, the time-varying wavelet must be suppressed effectively before the post-stack migration, so as to imaging accurately.

\section{CONCLUSIONS}

From the above analyses we can see that the time-varying nature of seismic wavelet has significant impact on every link of seismic data processing. Existing methods try to suppress or eliminate the time-varying wavelet from different perspectives, aimed to different links. However, the methods for the link of amplitude compensation and pre-stack deconvolution couldn't gain good effect because of many interfering factors in prestack seismic data, and accurate post-stack migration imaging is required that the wavelet is time-invariant. Therefore, poststack deconvolution is very important. If effective means are adopted to suppress the time-varying wavelet in post-stack deconvolution, the shortages of the front links can be made up for and the condition of imaging accurately can be met.

So it can be concluded that proposing more effective methods of time-varying seismic wavelet extraction and timevarying deconvolution in post-stack deconvolution will be the research emphases in seismic data processing in the future.

\section{REFERENCES}

[1] L.L. Wang, J.H. Gao, Z. Ming. A method for absorption compensation based on adaptive molecular decomposition. Applied Geophysics, 2010, 7(1), pp. 74-87.

[2] M.M. Zhang, Y.S. Dai, Y.N. Zhang, J.J. Ding and R.R. Wang. Timevariant seismic wavelet estimation method based on spectral modeling in time-frequency domain. Geophysical Prospecting for Petroleum, 2014, 53(6), pp. 675-682.

[3] J.W. Cheng, H.M. Gu, Z.Y. Xu. Simulation of explosion source wavelet in the actual formation. Chinese Journal of Engineering Geophysics.2009, 6(5), pp. 529-534.

[4] G.F. Margrave, M.P. Lamoureux, D.C. Henley. Gabor deconvolution: estimating reflectivity by nonstationary deconvolution of seismic data. Geophysics, 2011, 76(3), pp. W15-W30.

[5] G. Failla, M. Pappatico, G.A. Cundari. A wavelet-based spectrum for non-stationary processes. Mechanics Research Communications, 2011, 38(5), pp. 361-367.

[6] D. Hale. Q-adaptive deconvolution:Stanford Expl. Proj(SEG), 1982,30, pp. 133-158.

[7] S. Sinha, P.S. Routh, P. Anno. Instantaneous spectral attributes using scales in continuous-wavelet transform. Geophysics, 2009, 74, pp. WA137-WA142.

[8] R.G. Stockwell. A basis for efficient representation of the S-transform. Digital Signal Processing, 2007, 17(1), pp. 371-393.

[9] Mirko van der Baan. Bandwidth enhancement: Inverse Q filtering or time-varying Wiener deconvolution?. Geophysics, 2012, 77(4), pp. V133-V142.

[10] Mirko van der Baan. Time-varying wavelet estimation and deconvolution by kurtosis maximization. Geophysics. 2008, 73(2), pp. 11-18.

[11] L.L. Wang, J.H. Gao, W. Zhao and X.D. Jiang. Enhancing resolution of non-stationary seismic data by molecular-Gabor transform. Geophysics, 2012, 78(1), pp. V31-V41.

[12] C.Y. Qi, Y.P. Li, G.L. Zhang, J.X. Peng, G.L. Zhang and Y.B. Zhang. An improved generalized S-transform. Oil Geophysical Prospecting, 2010, 45(2), pp. 215-218.

[13] A.L. Rosa and T.J. Ulrych. Processing via spectral modeling. Geophysics, 1991, 56(8), pp. 1244-1251.

[14] B.W. Tang, B. Zhao, Y.H. Wu and H.Q. Li. An improved spectral modeling deconvolution. SEG Expanded Abstracts, 2010, 45(1): pp. 3668-3671. 